International Journal of Pure and Applied Mathematics

Volume 85 No. $4 \quad 2013,813-817$

ISSN: 1311-8080 (printed version); ISSN: 1314-3395 (on-line version)

url: http://www.ijpam.eu

doi: http://dx.doi.org/10.12732/ijpam.v85i4.15

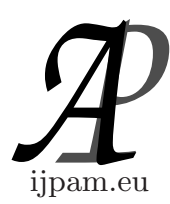

\title{
DERIVATIVE APPROXIMATION USING \\ A DISCRETE DYNAMIC SYSTEM
}

\author{
Paolo Mercorelli \\ Institute of Product and Process Innovation \\ Leuphana University of Lueneburg \\ Volgershall 1, D-21339 Lueneburg, GERMANY
}

\begin{abstract}
Derivatives of signals generate spikes if noise is present. This is the typical case in practical applications. This paper deals with an approximation of a derivative using a dynamic system to avoid spike and noise due to the calculation of this derivative. After formulating the problem, a proposition allowing to find a possible solution, which consists of the wanted approximation, is proven.
\end{abstract}

AMS Subject Classification: 26A24, 93C55, 49K15

Key Words: derivatives, discrete-time systems, computational methods

\section{Introduction and Motivation}

Derivatives and structures using derivatives, as for instance PD controllers, are very often used in industrial applications. Such kind of controllers are much used in practical applications of any nature. They are used also in electromagnetic valve control, [1] and more recently [2], [3] and [4]. This contribution emphasizes some mathematical aspects of an algorithm which the author used in practical applications such as for instance in [5] and in [6]. In particular, in [5] this algorithm is used in designing a velocity observer in the context of a throttle valve control. In [6] a similar algorithm is used to estimate the param-

Received: April 25, 2013

(c) 2013 Academic Publications, Ltd. url: www.acadpubl.eu 
eters identification in an application in which a synchronous motor is proposed. This paper proposes an approximated derivative structure to be taken into account for such kind of applications so that spikes, noise and any other kind of undesired signals which occur from the derivatives can be reduced. After the problem formulation this paper proves a proposition which allows to build this possible approximation of the derivative using a dynamic system. The paper is structured as follows. In Section 2 the problem formulation and a possible solution are proposed. At the end a remark discusses the results and the conclusion closes the paper.

\section{An Approximated Derivative Structure}

Using the derivative structures, imprecision occurs. The imprecision is due to spikes generating power dissipation. The idea is to find an approximated structure of general derivatives as they occur in mathematical calculations which are often used also in technical problems as proportional derivative controllers. The following formulation states the problem in a mathematical way.

Problem 1. If the following differential equation is given:

$$
r(t)=k_{p} e_{y}(t)+k_{d} \frac{d e_{y}(t)}{d t}
$$

where $e_{y}(t)=y_{d}(t)-y(t)$, and $y_{d}(t)$ and $y(t)$ are the desired and achieved position trajectories, respectively. In particular, $t$ represents the time variable with $t \in \mathbb{R}, y_{d}(t)$ and $y(t) \in \mathbb{R}$. The aim of the proposed approach is to look for an approximating controller $\hat{r}\left(t, e_{y}(t), k_{p}, k_{d}, k_{a p p}\right)$, where $k_{p}$ and $k_{d}$ are constants which are independent on differential variables. $k_{a p p}$ is a parameter, such that:

$$
\lim _{k_{a p p} \rightarrow+\infty} r(t)-\hat{r}(t)=0 .
$$

Proposition 1. The dynamic system

$$
\begin{aligned}
\frac{d \eta(t)}{d t} & =-\frac{k_{a p p}}{k_{d}} \eta(t)+\frac{k_{a p p}}{k_{d}}\left(k_{p} e_{y}(t)-k_{a p p} e_{y}(t)\right) \\
\hat{r}(t) & =\eta(t)+k_{a p p} e_{y}(t)
\end{aligned}
$$

where function $\eta(t) \in \mathbb{R}$, solves the problem defined above.

Proof. Considering the following approximate dynamic system:

$$
\frac{d \hat{r}(t)}{d t}=-\mathcal{M}\left(\hat{r}(t)-k_{p} e_{y}(t)-k_{d} \frac{d e_{y}(t)}{d t}\right),
$$


where $\mathcal{M}$ can be a function of $e_{y}(t)$ or a parameter with $\mathcal{M} \in \mathbb{R}$. If

$$
e_{r}(t)=r(t)-\hat{r}(t)
$$

then

$$
\frac{d e_{r}(t)}{d t}=\frac{d r(t)}{d t}-\frac{d \hat{r}(t)}{d t}
$$

If $\left\|\frac{d r(t)}{d t}\right\| \ll\left\|\frac{d \hat{r}(t)}{d t}\right\|$, then:

$$
\frac{d e_{r}(t)}{d t}=-\frac{d \hat{r}(t)}{d t}=\mathcal{M}(\hat{r}(t)-r(t))=-\mathcal{M} e_{r}(t)
$$

and $\mathcal{M}$ can be taken, so the system

$$
\frac{d e_{r}(t)}{d t}+\mathcal{M} e_{r}(t)=0
$$

is asymptotically stable. For guaranteeing the asymptotical stability,

$$
\mathcal{M}>0 .
$$

Also, for guaranteeing $\left\|\frac{d r(t)}{d t}\right\| \ll\left\|\frac{d \hat{r}(t)}{d t}\right\|, \mathcal{M} \gg 0$. A supplementary variable is defined as:

$$
\eta(t)=\hat{r}(t)-\mathcal{N}\left(e_{y}(t)\right)
$$

where $\mathcal{N}\left(e_{y}(t)\right)$ is a function to be designed with $\mathcal{N}\left(e_{y}(t)\right) \in \mathbb{R}$.

Considering that:

$$
\frac{d \eta(t)}{d t}=\frac{d \hat{r}(t)}{d t}-\frac{d N\left(e_{y}(t)\right)}{d t}
$$

let

$$
\mathcal{M} k_{d} \frac{d e_{y}(t)}{d t}=\frac{d N\left(e_{y}(t)\right)}{d t}=\frac{d N\left(e_{y}(t)\right)}{d e_{y}(t)} \frac{d e_{y}(t)}{d t} .
$$

If $\mathcal{N}\left(e_{y}(t)\right)=k_{\text {app }} e_{y}(t)$, then $\mathcal{M}=\frac{k_{a p p}}{k_{d}}$. If $k_{d}>0$, then the asymptotical stability is always guaranteed for $k_{a p p}>0$ and the rate of convergence can also be specified by $k_{a p p}>0$. From (7),

$$
\hat{r}(t)=\eta(t)+k_{a p p} e_{y}(t),
$$

and substituting (10) into (4), it follows (3). 
Remark 1. The proposed controller satisfies condition (2). Condition stated in equation (3) satisfies the following relation: $\frac{d e_{r}(t)}{d t}=\frac{d r(t)}{d t}-\frac{d \hat{r}(t)}{d t}$, where $e_{r}(t)=r(t)-\hat{r}(t)$. If assumption $\left\|\frac{d r(t)}{d t}\right\| \ll\left\|\frac{d \hat{r}(t)}{d t}\right\|$ is satisfied. This assumption is a technical one and it is used to simplify the proof of Proposition 1. A possible method to prove Proposition 1 removing this assumption is the singular perturbation approach which in presented in [7].

Using Euler method for the discretization of the controller described in (3), this structure follows:

$$
\begin{aligned}
\eta(k) & =\frac{\eta(k-1)}{\left(1+t_{s} \frac{k_{a p p}}{k_{d}}\right)}+\frac{t_{s} \frac{k_{a p p}}{k_{d}}\left(k_{p}-k_{a p p}\right) e_{y}(k)}{\left(1+t_{s} \frac{k_{a p p}}{k_{d}}\right)} \\
u(k) & =\eta(k)+k_{a p p} e_{y}(k),
\end{aligned}
$$

where $u(k)=\hat{r}(k), t_{s}$ indicates the fixed sampling time with $t_{s} \in \mathbb{R}$ and $k$ represents the discrete index of the discrete variables with $k \in \mathbb{N}$. After the transformation of the controller shown in (11) with the Z-transform, these equations are obtained:

$$
U(z)=\frac{\left(t_{s} \frac{k_{a p p}}{k_{d}}\left(k_{p}-k_{a p p}\right)\right) E_{y}(z)}{\left(1+t_{s} \frac{k_{a p p}}{k_{d}}\right)-z^{-1}}+k_{a p p} E_{y}(z),
$$

and

$$
U(z)=\frac{\left(t_{s} \frac{k_{a p p}}{k_{d}} k_{p}+k_{a p p}-k_{a p p} z^{-1}\right) E_{y}(z)}{\left(1+t_{s} \frac{k_{a p p}}{k_{d}}\right)-z^{-1}} .
$$

In expressions (12) and (13) $z \in \mathbb{C}$ and represents the well known complex variable.

\section{Conclusion}

This paper deals with an approximation of a derivative using a dynamic system to avoid spikes and noise. After formulating the problem, a proposition allowing to find a possible solution, which consists of the wanted approximation, is proven. 


\section{References}

[1] C. Tai, A. Stubbs, T. Tsao, Modeling and control design of an electromagnetic engine valve, In Proceedings of the American control conference, Arlington, Va (2001).

[2] P. Mercorelli, A two-stage augmented extended kalman filter as an observer for sensorless valve control in camless internal combustion engines, IEEE Transactions on Industrial Electronics, 59, No. 11 (2012), 4236-4247.

[3] P. Mercorelli, An anti-saturating adaptive preaction and a slide surface to achieve soft landing control for electromagnetic actuators, IEEE/ASME Transactions on Mechatronics, 17, No. 1 (2012), 76-85.

[4] P. Mercorelli, A hysteresis hybrid extended kalman filter as an observer for sensorless valve control in camless internal combustion engines, IEEE Transactions on Industry Applications, 48, No. 6 (2012), 1940-1949.

[5] P. Mercorelli, Robust feedback linearization using an adaptive pd regulator for a sensorless control of a throttle valve, Mechatronics a journal of IFAC. Elsevier publishing, 19, No. 8 (2009), 1334-1345.

[6] P. Mercorelli, A decoupling dynamic estimator for online parameters identification of permanent magnet three-phase synchronous motors, In: Proc. 16-th IFAC International Symposium on System Identification 2012, Brussels (2012), 757-762.

[7] H.K. Khalil, Nonlinear Systems, Prentice Hall (2002). 
